the forest-trees to-day? The records are silent upon these points. A discovery that I made in the vicinity of Tokio last year leads me to believe that possibly the traces of a race of men previous to the Aino occupation have been found." Again I say : "The next question arises as to whether the deposits are Aino or pre-Aino. The race who left these remains were pot-makers par excellence. It is generally admitted by ethnologists that the art of pottery once gained is never lost. It is a fact, however, that neither the Esquimaux, Aleutians, Kamtchadales, nor the Ainos are essentialy earthen pot makers." And, again, having shown incontestible proofs of the evidences of cannibalism in these deposits, I ask, "Were the Ainos cannibals? Repeated inquiries among eminent Japanese scholars and archæologists, like Mr. Kanda, Mr. Ninagawa, and others, as to this question, are always answered in the same way. Not only were they not cannibals, but they are reported as being so mild and gentle that murder was never known to have occurred. So monstrous a habit would certainly have been known and recorded, particularly in the painstaking annals of early historians."

In the Proceedings of the American Association for the Advancement of Science for 1878 occurs in the list of papers read by title the following one of mine, entitled "Evidences of Cannibalism in a Nation before the Ainos in Japan." A foot note states that this paper was published in the Tokio Times

In the year 1879 the University of Tokio published my memoir on the "Shell Mounds of Omori," illustrating the various forms of pottery, bone implements, etc., by seventeen folded plates. While this memoir is devoted exclusively to a minute description of the Omori deposits as a basis of comparison with material that I had on hand for the description of other shell-heaps, yet I urged the evidence of the deposits not having been made by Ainos, but by a race anterior to the Ainos, and cited especially the evidences of cannibalism as bearing on this point.

Twelve years ago I had occasion to criticise and controvert (American Naturalist, September, 1880), in the most emphatic manner Professor Milne's views as published in the Transactions of the Asiatic Society of Japan. At the same time I also showed, as I believed, the fallacy of the views of Henry von Siebold on this question. Thus in various publications in $1877,1878,1879$, and $1880 \mathrm{I}$ have urged the existence of a pre-Aino race in Japan.

Had Mr. Hitchcock taken the trouble to give proper credit to others who had worked in this field, he would have found additional support to the position he takes; as it is, his paper is marred by misapprehension and by the injustice of these omissions.

Salem, Mass., Aug. 30. EDWARD S. MORSE.

\section{On the Fundamental Hypotheses of Abstract Dynamics;} From Another Point of View.

THERE is at present very little agreement among physicists or philosophers as to the nature of the hypotheses or laws upon which dynamics is based. On Aug. 5 Professor MacGregor expounded one view of the matter in these columns; but as I cannot but think his view contains some logical imperfections, I wish to lay before your readers a different view with which to compare it. For this is not a question to be settled by authority; the arguments on either side are after all simple enough, and, having studied them, any man of average attainments is capable of weighing them and forming his own opinion.

The principles of abstract (subjective) geometry may be deduced from definitions of the terms "Position" and "Direction," gether with certain axioms asserting the conceivability of geometrical figures and constructions. Even without these axioms a symbolic geometry might be deduced, whose conclusions, however, would be mere truisms, or verbal assertions, till they were given a meaning by the axioms. To proceed to the objective geometry of material space, we require in addition certain inductions; which, however, are so complete that no practical doubt remains as to their validity.

1 See my "Foundations of Geometry," Deighton, Bell, \& Co., Cambridge, Eng., 1891.
In the same way we may treat kinematics from three different points of view. Symbolically, it is sufficient to define Time implicitly by the assertion, "The positions of points are all continuous single-valued functions of the Time." This definition may be given a subjective meaning by the axiom, "Particles are conceivable in Time," and an objective meaning by an induction proving that "material particles exist only in Time," i.e., their positions are continuous single-valued functions of a certain variable, which we may call Time.

To proceed to kinetics symbolically, we require definitions of Mass and Force. The only connotation symbolically required for the former term is "Mass is not a function of Space or Time." The latter term may be defined implicitly by assertions equivalent to Newton's laws of motion, which may be stated thus :--

1. The resultant force on any particle in any direction, referred to a given set of axes, is the product of the measures of its mass and its acceleration in that direction.

2. All forces go in pairs between pairs of particles, equal forces in opposite directions acting on the particles respectively in the line joining them. (Such a pair of forces may be spoken of as a stress.)

It is evident from 1, since mass is not a function of space or time, that forces, like accelerations, are vectors, and may be compounded by the parallelogramic law. Paragraph 1, however, only speaks of resultant forces, and the actual, or acting, forces on any particle would remain entirely arbitrary but for paragraph 2 , which must be read in conjunction with 1 . Professor MacGregor asserts that paragraph 2 is not consistent (i.e., might be inconsistent) with 1 . So far from this being the case, I propose to show that it still leaves the term Force to some extent arbitrary. The stresses between particles are not completely determined, even with reference to a given set of axes; and, moreover, both Force and Stress are relative to the axes chosen.

In geometry and kinematics both position and direction are relative terms. To determine a position we require to know its distance and direction from a given position. To know its direction we require to know the inclination of that direction to two given (independent) directions, and, in addition, which side it is of the plane determined by them.

Suppose, then, we have a set of particles numbered from 1 to $n$. Choose the first particle as origin of a system of rectangular coordinates; the direction 12 as that of the axis of $x$; the direction at right-angles to this in the plane 123 , and on that side of the line 12 on which the particle 3 lies, as that of the axis $y$; and the direction perpendicular to the plane 123 , on that side of it on which the particle 4 lies, as that of the axis $z$. Thus we have determined a set of axes completely, and in doing so we have made the six arbitrary assumptions:-

$$
\left.\begin{array}{ccc}
x_{1}=0 & y_{1}=0 & z_{1}=0 \\
& y_{2}=0 & z_{2}=0 \\
& & z_{3}=0
\end{array}\right\}
$$

Now let $F_{r s}$ be the stress between the particles $r$ and $s$, being positive if they attract, negative if they repel one another. Then considering forces asting on particle 1 we have the equations -

$$
F_{12} \frac{x_{1}-x_{2}}{r_{12}}+F_{13} \frac{x_{1}-x_{3}}{r_{13}}+\cdots--m \ddot{x}_{1},
$$

and two similar equations with $y$ and $z\left(r_{12}\right.$ being the distance between the particles). Thus in all we have $3 n$ equations between $\frac{n-n-1}{2}$ quantities $F_{12}, F_{13}$, etc. But these equations may not all be independent. As, however, they contain $(3 n-6)$ independent variables, $x_{2}, x_{3}, y_{3}$, etc. (the other six having been arbitrarily equated to zero), there will in general be $(3 n-6)$ of them independent. If they only just sufficed to determine the quantities $F_{12}, F_{13}$, etc., we should have

$$
\frac{n-n-1}{2}=3 n-6 \text {. }
$$

Whence $n=3$ or 4 . Therefore, if $n$ is greater than 4 (which, of course, it is), the equations must be insufficient to determine the quantities; that is, the stresses remain to some extent arbitrary; 
hence the two assertions about Force are arbitrary and may be laid down as a (partial) definition of that term.

From this definition all the theorems of dynamics may be deduced, as from Newton's laws of motion. The theorems of statics may also be deduced, the only difficulty being the principle of virtual work. This difficulty, however, disappears as soon as the term "geometrical conditions" is properly defined.

We have then a symbolic dynamics, To give it a subjective meaning we have to conceive a real denotation for its terms. It is not, however, necessary to give a real denotation to Force if we can do so to Mass, for we may still regard Force as merely a nam $\theta$ for the product of mass by acceleration, or (which is the same thing) as the time-flux of momentum. To give the theory an objective application it is necessary to show that what we call material particles not only occupy positions which are continuous one-valued functions of what we call Time, but also possess a certain characteristic which is not a function of space or time, and which may be called Mass. Then, whether we attach any denotative meaning to Force or not, we can discuss the forces or stresses that must be postulated between various particles of matter. The magnitudes of these will in general depend on the axes we assume by which to determine positions, and also on the masses assigned to the various particles. The axes and masses are therefore assumed in such a way as to make the resulting system of stresses the simplest possible. For example, it is generally assumed that the stress between any two particles diminishes as the distance between them increases, and may be neglected if this distance is very great. Hence in astronomy the attractions of the fixed stars on the planets may generally be neglected, and we may discuss the solar system alone. It is further shown that the system of stresses between the sun and planets is simplest when a certain plane is taken as "the invariable plane." But we do not really know that the stresses thus deduced are the actual ones, or indeed that there is any actual phenomenon corresponding to what we call stress at all. Any plane might be chosen as the "invariable" one, at the cost of having to postulate a more complicated system of stresses. We cannot determine fixed directions dynamically, any more than kinematically, except by making assumptions which are really arbitrary about the stresses between certain particles.

As Professor MacGregor points out, the law of the conservation of mechanical energy would flow from the assumption that stresses are functions of the distances between the particles on which they act. But this would not include the general law of conservation of energy until all energy was shown to be mechanical energy. And even then, on the above assumption, the term conservation of energy would be rather misleading; for the kinetic energy is not conserved unless the term potential energy is merely used as a cloak to hide our ignorance of kinetic energies which for the moment have passed beyond our ken. For example, a few years ago it might have been said that when we project a keeper away from an electro-magnet, the kinetic energy with which it starts becomes converted into potential by the time it stops, just as when we throw a stone into the air. But if, while the keeper is at a distance from the magnet, the current is switched off, that potential energy is abolished! The true view is, however, that there never was any potential energy at all, the energy of the flying keeper had its equivalent in an increase in the electric current round the magnet - a kinetic, not a potential, energy. And I have no doubt that some day science will show a similar explanation to hold with respect to gravitation and other actions at a distance. When that day comes the term "potential energy" may be banished to "the limbo of once useful things."

It will be seen, therefore, that I differ from Professor MacGregor chiefly in denying "the non-relative character of Force." Professor MacGregor says, "it is easy to show that if it [the third law of motion] hold for one point of reference, it cannot hold for another having an acceleration relative to the first." I should like to see his proof; but if he refers accelerations to a single point, I can well understand that he should arrive at results inconsistent with mine. For, as I have shown, the apparently absolute determinations of direction depend in reality on arbitrary assumptions as to stresses. Having made these arbitrary assumptions, it may well be impossible to further make arbitrarily the assumptions involved in the third law of motion.

I cannot quite follow his paragraph beginning " It may easily be proved that the stress between two particles is proportional to the product, by the sum of their masses into their relative acceleration." There seems to be some misprint; but how a single particle could in any case exert all the forces acting on a system of particles, I cannot understand, unless the words "equal and opposite" in the third law of motion are not held to imply that the forces act in the line joining the particles, which, moreover, is distinctly implied in the Professor's law of stress. In any case the difficulty referred to above comes in again, viz., that we cannot determine directions absolutely, or positions by reference to a single point.

In conclusion, I should like to point out that it seems inconvenient, even if Professor MacGregor's views be accepted on other points, to include in one law of stress, two statements resting on such very different evidence as that forces may be considered to be attractions or repulsions, and that their magnitudes depend solely on the distances between the particles on which they act. It would give a student a very false notion of the fundamental hypotheses of dynamics to teach him that he must accept or reject both these assertions together.

EDWARD T. DIXON.

Cambridge, Eng., Aug. 20.

\section{The Fundamental Hypothesis of Abstract Dynamics.}

Professor Hoskins points out (Science, Aug. 26, p. 122) that for the conservation of energy the necessary and sufficient condition is that $\Sigma P d r$ shall be a perfect differential of a function of the quantities $r, P$ being the stress between any two particles of the system, and $r$ their distance; and that the condition that each $P$ shall be a function of the corresponding $r$ only, which I suggested for adoption as a fourth law of motion, with a view to the deduction of the law of the conservation of energy (Science, Aug. 5, p. 74), while sufficient, is not necessary.

There are three reasons which influence me in selecting for the fourth law an hypothesis which is more than sufficient for the main purpose in making the selection, viz., (1) that it is capable of simple physical expression, (2) that it is already known to hold in the case of several natural forces, and (3) that the additional assumption involved in it, over and above that necessary for the deduction of the conservation of energy, is one which is, I think, invariably made in investigations on the laws of natural forces.

What the additional assumption is, is readily seen. In a system of two particles $A$ and $B, \Sigma P d r$ becomes $P d r$; and in this case it is both necessary and sufficient for the conservation of energy that the single stress acting shall be a function of the distance $A B$ only. If we add a third particle, $C$, to the system, conservation no longer requires that the stress between $A$ and $B$ shall be a function of the distance $A B$ only, though it is secured if that condition is fulfilled. Thus the proposed law assumes, in addition to what is required for conservation, that the stress between $A$ and $B$ is not changed by the fact that other stresses have begun to act between $A$ and $C$ and between $B$ and $C$. The proposed law therefore involves an assumption similar to that implied in Newton's second law. As Newton's law assumes that a force produces the same acceleration in a particle whether other forces act on it or not, so the proposed law assumes that the stress between two particles is the same whether or not there are other stresses acting between them and other particles.

That this additional assumption holds in the case of some natural forces has been abundantly verified, and in investigations into the laws of forces not yet determined, so far as my knowledge of such investigations goes, the same assumption is always made. This being so, we would seem to be warranted in adopting, tentatively of course, as a fourth law of motion an hypothesis in which this assumption is implied. The proposed law cannot be said to have received anything like the verification that Newton's laws have received. But of the many deductions which have been made from it, none have been contradicted, while many have been corroborated, by experience.

Shubenacadie, N.S., Sept. 2. 